immer geneigt gewesen, den Exhalationen von Vulcanen zuzuschreiben. Jetzt hat er diese Ansicht in dem Vor. kommen des Hayesins in Chile bestätigt gefunden. Dieses Mineral bildet nämlich schneeweisse seideglänzende Flocken, welche in den heissen Quellen, genannt Baños de Toro (Stierbäder), der Cordilleren bei Coquimbo suspendirt sind und schliesslich sich absetzen. Augenscheinlich geht ihre Bildung so vor sich: die heissen Dämpfe, welche ohne Zweifel den benachbarten Vulcanen entstammen, dringen durch Canäle und Spalten, mit Borsäure beladen, in jenes Quellwasser, welches ans den sich zersetzenden Kalkfeldspathen der Porphyrtuffe und oolithischen Sandsteine jener Gegend eine reichliche Menge Kalk aufgelöst hat. Auf ähnliche Weise wird auch der in den toscanischen Lagunen sich findende Hayesin entstanden sein.

\title{
III.
}

Die Feuerbeständigkeit der Thone nach den Resultaten synthetischer Versuche, analytischer Untersuchungen und der Erfahrung in technischer wie mineralogischer Beziehung.

Von

Dr. Carl Bischof.

Die drei angedeuteten Wege sind es, welche zur Ergründung der vorliegenden, vielfach angeregten und bearbeiteten, aber dennoch dunklen Frage führen möchten: der synthetische, analytische und der auf die praktische Erfahrung begründete.

Die sogenannten flussbildenden Bestandtheile, deren Menge allerdings eine gewisse Grenze, welche bei übrigens 
ausgezeichnet strengfü̈ssigen Thonen durchats keine engbeschränkte ist, nicht ubberschreiten darf, sind es keineswegs, wonach man einzig und zuerst bei Beurtheilung der Güte eines feuerfesten Thones zu fragen hat, wiewohl unter Anderen Gaffard ein Verfahren „nichtfeuerfeste Thone in feuerfeste umzuwandeln" und Bower ein Patent darauf begründet hat*).

In einem Thone kann die Menge der beigemengten Alkalien, des Eisens und Kalks vorhältnissmässig selbst sehr wenig betragen und doch gehört der betreffende Thon nicht zu den strengflüssigen. So z. B. finden sich Thone, wie ich einen solchen, vorkommend auf der preussisch-belgischen Grenze unweit Aachen, analysirte, dessen flussbildende Bestandtheile im Mittel von zwei genauen Bestimmungen nur 1,80 p.C. betragen, und doch ist seine Strengflüssigkeit $=$ nalıezu 5 , d. h. er ist kaum noch den gewöhulichen feuerfesten Thonen zuzuzählen. Andererseits giebt es unter anerkannt höchst strengflüssigen, englischen wie belgischen Thonen, solehe, bei denen die flussbildenden Bestandtheile in Summe selbst bis zu 5 p.C. ausmachen, wobei uns jedoch, beiläufig erwähnt, das bemerkenswerthe Verhältniss entgegentritt, dass derartige Thone wenig Alkalien enthalten. Dann wirken, worauf unter Anderen Asmus in seinen praktischen und trefflichen Aufsätzen ${ }^{* *}$ ) hingewiesen hat, diese Flussmittel verschieden nachtheilig. Am nachtheiligsten sind, wie Zi i rek treffend auseinander gesetzt hat, die Alkalien, dann dürfte folgen das Eisen, namentlich das Eisenoxydul, hicrauf die Kalkerde, und am wenigsten nachtheilig ist die Bittererde.

Es stimmt dicse in Allgemeinen erfahrungsmäissig bcgründete Ordnung überein mit der Schmelzbarkeit der Verlindungen dieser Basen mit Kieselsäure, deren relatives Verhältniss ich früher zu ermitteln suchte ${ }^{* * *}$ ) und woraus hervorgeht, dass eventuell, wenn es an Kieselsäure fehlt, um ein Eisentrisilicat zu bilden, das Eisen entschieden nachtheiliger wirken muss, als die Kalkerde.

*) Dingler's Polytechn. Journ. CIV, 398 und CLXII, 239.

**) Berggeist, 1559 , Nr. 39 u. 40

***) Polytechn. Jonrn. CLXV, 378 . 
Fast genau steigt die Strengflüssigkeit der Silicate mit der Zunahme der Sättigungscapacitäten der Basen.

Ist sowohl die Quantität als Qualität der flussbildenden Bestandtheile nur bedingt maassgebend, so müssen andere Verhältnisse um so unbedingter entscheidend sein.

Ziehen wir die Hauptbestandtheile eines jeden Thones: die Kieselsäure und die Thonerde, und deren Verhältniss zu einander in Betracht, so hat man wohl ron der Voraussetzung der sehr grossen Strengflïssigkeit der Kieselerde an sich geschlossen, dass mit deren verhältnissmässiger $\mathrm{Zu-}$ nahme auch die Strengfluissigkeit der Thone zunehme. Auch der praktische Versuch schien cine solche Voraussetzung zu bestätigen, da allerdings die meisten Thone durch Zusatz von Quarzsand strengflüssiger zu machen sind, was täglich fabrikmässig geschieht, und steht diese Erfahrung ja in genanem Zusammenhang mit der Erhöhung der Strengflüssigkeit der verschiedenen Silicate durch Vermehrung der Kieselsäuremenge, wie z. B. die Trisilicate der resp. Frden, strengflüssiger sind, als dic Monosilicate. Das Gemenge mit dem grösseren Kieselsäure- resp. Quarzzusatz braucht längere Zeit der Erhitzung, ehe die Silicatbildung - sofern man darunter das Uebergehen des pulvrigen, losen Zustandes in den festen, dichten mit wenigstens oberflächlichem Glasüberzug versteht - eintritt, als das mit dem geringeren, so dass man gewissermaassen aus dem Grade oder der Zeit der Schmelzbarkeit, die Kieselungsstufe eines Silicats erkennen kann.

Diese Unterschiede treten deutlich hervor bis zu der Temperatur, welche die Silicatbildung bedingt; wird aber die Temperatur höher gesteigert, so machen sich andere Verhältnisse geltend. Wird nämlich die Prüfungshitze erhöht über den Schmelzpunkt des Gusstabls bis zu dem des Schmiedeeisens oder noch höher, so dass die Silicatbildung bereits eingetreton, so ist umgekehrt das gebildete Silicat ein molr flüsiges, wenn die Kicselerdo vorwallender ist.

Mit anderen Worten: wir müssen zwischen der erforderlichen Temperatur zur Billung der Thonsilicate und derjenigen zum Flüssigwerden der gebildeten Silicate unterscheiden. 
Die Bildung verlangt eine um so länger andauernde Erhitzung, je mehr Kieselerde in die Verbindung eingehen soll; das Flüssigwerden der ganzen Masse der gebildeten Silicate, wozu überhaupt eine höhere Temperatur erforderlich, findet umgekehrt, eher und vollständiger statt bei vorherrschender Kieselsäure.

Man hat in neverer Zeit, wie Fresenius in seinen unübertrefflichen Thonuntersuchungen ausführte, unterschieden zwischen der chemisch gebundenen Kieselsänre und dem mechanisch beigemengten Quarzsande, was freilich keineswegs unwichtig ist, und worauf ich weiter unten noch zurückkommen werde: man hielt einen Thon für um so strengflüssiger, je mehr Kieselsïure im gebundenen Zustande darin enthalten ist. Auch hierfür gilt im Ganzen das eben Gesagte und lässt sich diese Behauptung, so allgemein ausgesprochen, nicht begriinden.

\section{Synthetische Versuche.}

Mengt man 1 Theil chemisch reines Thonerdepulver mit 1,801 chemisch reinem Quarzpulver zusammen, worin die Sauerstoffmengen - nach der Formel $\mathrm{Al}_{2} \mathrm{O}_{3}, 2 . \mathrm{SiO}_{3}-$ gleich oder neutral und somit das Gemenge dem Monosilicat entspricht, und ebenso 1 Theil Thonerde mit 3,601 und 5,402 Quarz, welche Gemenge das Bi- und Trisilicat geben, und setzt dieselben, zu kantigen Prismen geformt, einer heftigen Glïhhitze aus, welche über den Schmelzpunkt des Gusstahls geht und den des Schmicdeeisens erreicht, so beginnt zuerst das Monosilicat mit einer glasigen Rinde sich zu überziehen und erst spater das Bi- und Trisilicat, welche letztere fast gleichzeitig zu schmclzen anfangen.

Zur Controlirung des Hitzegrades wurde ein kantiges Stiick Schmiedeeisen in eine Platinkapsel eingeschlossen, welcher als Unterlage ein vertieftes Tellerchen aus reiner Thonerde diente. Das Eisenstiickchen rundete sich ab mit völlig convexen Flächen. An der Stelle, wo das Schmiedeeisen das Platin berührte, war die Kapsel, welche übrigens ungeschmolzen war, stets ein wenig zerfressen. 
Vermindert man dagegen die Menge der Kieselsäure und mengt 1 Theil chemisch reines Thonerdepulver mit 0,9 - nach der Formel $\mathrm{Al}_{2} \mathrm{O}_{3}, \mathrm{SiO}_{3}$ - und ebenso 1 Theil Thonerde mit 0,45 und 0,22 chemisch reinem Quarzpulver und setzt diese Gemenge im Vergleich zu dem Mono-, Biund Trisilicate dem bezeichneten Feuergrade aus, so dass letztere sich mit einer glasigen Rinde überziehen, so bieten sich bei den basischen Thonsilicaten keine Zeichen von Schmelzung dar. Während erstere eine aussen glasartigglänzende Masse bilden, zeigen letztere ein glanzloses, mattes Aeussere. Steigert man den Hitzegrad noch höher, so weit, als es Thontiegel aus dem bestbekanten Steinkohlenthon eben aushalten ohne zusammenzugehen, so geben sich auf Grund verschiedentlich abgeänderter und wiederholt bestätigter Versuche, folgende Schmelzverhăltnisse zu erkennen*).

Das dreifach-basische Thonsilicat zeigt keine Glasirung und keinen Glanz weder äusserlich noch auf der Bruchfläche. Die Masse ist durchaus körnig; doch sind die Gemengtheile ziemlich fest zusammengebacken. - Das zweifach- wie einfach-basische Gemenge verhält sich im Ganzen ähnlich; die Bruchflächen erscheinen etwas dichter und bei letzterem. mehr wie bei ersterem. - Das Monosilicat oder neutrale Silicat ist glasirt, durchscheinend. Die Form hat sich erhalten; der Bruch aber ist porcellanähnlich, wenn auch noch einigermaassen körnig. - Das Bi- und Trisilicat dagegen ist zusammengeflossen zu einem homogenen dichten Email, ohne im mindesten körnig zu sein. - Das Trisilicat beginnt porös zu werden, ein Zeichen grösserer Flüssigkeit.

Es sind demnach die basischen Thonsilicate entschieden strengfü̈ssiger als die sauren, und das neutrale, das Monosilicat, wird in einem sehr heftigen IItzegrade angenscheinlich weniger flissig, ist mithin feuerbeständiger als das Bi- und Trisilicat.

*) Der angewandte Hitzegrad lag weit über dem Schmelzpunkt des Schmiedceisens, er war blendendes Weissglühen, und ein solcher, dass ein Stück Platindraht, eingeschlossen in einc Kapsel aus chemisch reiner Thonerde, zur Kugel zusammenschmolz. Der Draht war bezogen von Heräus in Hanau und licss sich die Kugel aushämmern zu einem feincn Plättchen, ebenso wic der ungeschmolzene Draht, doch zeigte sich dasselbe mehr kantenrissig. 
Gleizeitig wurden demselben schr hohen Hitzegrade reine Thonerde und Kiesclerde, sowohl künstlich dargestellte als natürliche, so rein als beide in der Natur zu finden sind ausgesetzt.

Die Thonerde wurde dargestellt aus der in der Harburger Kryolithfabrik fabricirten schneeweissen Thonerde. Dieselbe wurde zur völligen Befreiung ron kohlensaurem Natron mittelst Salzsäure in der Platinschale gelöst, völlig zur Trockne eingedampft, mit Salzsäure befeuchtet und hieranf mit Wasser digerirt. Das Unlösliche wurde abfiltrirt, die Thonerde durch Ammoniak gefällt und bis zur 20000 fachen Verdünnung decantirt, dann aufs Filtrum gebracht, mit kochendem Wasser wiederholt ubbergossen und so ganz rein (nach der Uhrglasprobe) ausgewaschen; hiorauf getrocknet und endlich im Achatmörser pulverisirt. Die so gereinigte Thonerde, in Kalilauge gelöst und zur Trockne eingedampft, löste sich vollkommen klar in Salzsäure. Als natürliche Thonerde wurde genommen edler blauer Corund, Sapphir, wasserheller und ein smalteblauer.

Die Kieselerde wurde doppelt gereinigt. Ausgesuchte reinweisse Quarzstücke wurden nämlich im Achatmörser pulverisirt, dann längere Zeit in einem Glaskolben mit Salpeter-Salzsäure digerirt und ausgewaschen. Um eine etwaige Verunreinigung durch das Glasgefäss zu entfernen, wurde dieses Pulver nochmals in einer Platinschale mit Salzsäure andauernd digerirt und hierauf auf das sorgfältigste ausgewaschen, getrocknet und scharf geglüht. Die so zubereitete Kieselerde, mit Flussäure geprüft, liess nicht den mindesten Rückstand. - Als natürlicher Quarz wurde gew ählt cin besonders reiner, durchscheinender, norwegischer Rosenquarz; ferner völlig durchsichtiger, wasserheller Bergkrystall, und durchsichtiger, fast farbloser Amethyst.

Die Proben kamen, um jedwede fremdartige Beimischung durch die Unterlagen zu vermeiden, je einzeln zu liegen aur verticften Tellerchrn von stets derselben entsprechenden chemsch reinen Stibstanz, die auf das allerfoinste pulverisint, mit Wasser zu einom Toig nugemacht, geformt und alsdann vorher hifigl gegliuht waren bis zu einer losen Sinterung. - Die Silicate wurden demnach geprüft auf 
Tellerchen, wovon ein jedes aus der entsprechenden reinen Silicatmasse bestand, die Thonerdeproben auf solchen aus reiner Thonerde, und die Kieselerde auf solchen aus reiner Kieselerde. Die Tellerchen wurden auf einer Thonscheibe, aus dem besten kohlenfreien feuerfesten Thon gefertigt, aufgekittet und eingesetzt in einen gut geschlossenen kleinen Tiegel aus derselben besten Thonmasse.

So vergleichend diese Probẻprismen demselben, oben näher bezeichneten sehr hohen Hitzegrade, in mehrmals wiederholten Versuchen ausgesetzt, ergab sich:

Die chemisch reine Thoncrde ist ohne Zeichen einer glasigen Schmelzung, auch an den Kanten, die Masse ist aber beträchtlich geschwunden, hat sich verdichtet, ist fest und hart geworden wie der Bruch zeigte.

Der Corundsplitter ist dunkler von Farbe geworden, lässt aber sonst keine Veränderung oder irgend welche Merkmale von Schmelzung erkennen.

Die doppelt gereinigte Kieselsäure zeigt weder im Aeusseren noch Inneren der Probe Kennzeichen einer Schmelzung. Der Bruch ist körnig und wenig fest (ziemlich lose).

Die einfach gereinigte Kieselsäure zeigte sich schon schmelzbarer, sowie sofort sich deullich zu erkennen gab die grössere Leichtflüssigkeit, wenn dieselbe in einem Porcellanmörser und noch mehr, wenn sie in einem Glasmörser gerieben wurde.

Kieselerde, wie sie bei den Silicatanalysen nach dem Aufschliessen mit kohlensaurem Alkali erhalten wird, selbst auf das Allersorgfältigste mit kochendem Wasser längere Zeit ansgewaschen, verhielt sich gleichfalls weniger strengflüssig.

Der norwegische Quarz ist völlig glasirt, theils durchsichtige, theils milchweiss-blasige Glasmasse bildend.

Der wasserhelle Bergkrystall zeigt einon dïnnen glasigen Ueberzug (Schmelz), ohne abgerundeto (abgeflossene) Kanten; er ist weiss und undurchsichtig geworden.

Der Amethyst verhält sich ähnlich. In der mehr durchsichtigen Masse schwimmen vereinzelte weisse Flitter. 
Aus vorstehenden Versuchen geht demnach hervor, dass die mit Sorgfalt dargestellte chemisch reine Thonerde weniger strengflüssig ist als die chemisch reine Kieselerde; dagegen ist die natürliche reine Thonerde strengflüssiger; als die natürliche Kieselerde und zwar als der reine norwegische Rosenquarz, der Bergkrystall und Amethyst.

\section{Analytische Untersuchungen.}

Selbstverständlich lässt sich bei den äusserst verbreitet, aber stets unrein in der Natur vorkommenden Thonen, deren Fenerbeständigkeit meist gleichzeitig durch mehrere verschiedenartige Verhältnisse modificirt wird, eine geltende Gesetzmässigkeit besonders schwierig erkennen.

Bei einer Mannigfaltigkeit von Verhältnissen und Bedingungen überhaupt, lässt sich eben nur aus einem sich ergebenden Zusammenstimmen im Allgemeinen oder aus einem mittleren vorwiegenden Resultate, mit einer gewissen Sicherheit ein Schluss ziehen. Besteht eine Gesetzmässigkeit, so musste sie um so leichter sich zu erkennen geben bei einer Auswahl von Thonen, die von demselben Vorkommen und überhaupt einander alhnlich, aber dennoch hinsichtlich der Strengflüssigkeit verschieden sind.

Ich wählte zu dem Zweck sechs Thonsorten von Audenac in Belgien, sämmtlich vorkommend in der Umgegend von Namur an sechs verschiedenen Fundpunkten, mit denen vergleichungsweise der bestbekannte strengflüssige schottische Thon, der von Garnkirk, analysirt wurde.

Bekanntlich findet sich der feuerfeste belgische Thon im Uebergangskalke in Mulden, deren Durchmesser bis zu circa 200 Fuss steigt, und die circa 100 Fuss tief sind, nach unten gewöhnlich durch eine Sandschicht abgeschlossen und nach oben allmählich in gewöhnlichen Ziegelthon übergehend.

Der schottische Thon ist, wie bekannt, ein grauer, wenig bindender, kohlehaltiger Schieferthon, welcher sich in der Steinkohlenformation zu Garnkirk bei Glasgow findet und verschiedene Schichten von wechselnder Güte bildet. Der vorzüglichste feuerfeste Thon kommt in einer mittleren Schicht von 30 Zoll Mächtigkeit vor. 
Physikalische Eigenschaften. - Die untersuchten belgischen Thone gehören sämmtlich zu den fetten, die an der Zunge stark anhaften, im Wasser zerfallen unter Entwickelung kleiner mit Zischen entweichender Luftbläschen, und die damit angefeuchtet, eine sehr bindende, plastische Masse geben. - Sie fühlen sich fettig an. - Mit Säure übergossen brausen sie nicht, nur Nr. 2 ein wenig. - Ueber der Spiritusflamme geglüht, schwärzen sie sich, enthalten daher organische Reste.

In dem Achatmörser zerrieben, knirschen sie alle, Nr. 3 am meisten, in Folge einer Beimischung gröberer Sandkörnchen.

In der Farbe sind sie etwas verschieden von einander:

Nr. 1 ist von hell-schieferblauer Farbe, das Pulver bläulich-grau.

Nr. 2 ist von derselben nur ein wenig helleren Farbe.

Nr. 3 ist hell-blaugrau, das Pulver schmutzig-gelblichgrau.

Nr. 4 ist blaugran, das Pulver hell-blaugrau.

Nr. 5 ist von gleichfalls hell-blaugrauer Farbe, das Pulver gelblich-grau.

Nr. 6 ist gelblich-grauweiss und das Pulver ebenso.

Der Grad der Strengflüssigkeit wie des Bindevermögens der fraglichen Thone wurde auf Grund des in dieserm Journal (LXXXIV, 354) beschriebenen Verfahrens geprüft, wonach die Menge des chemisch reinen Quarzzusatzes das Maass für die Strengflüssigkeit des Thones in umgekehrtem und für das Bindevermögen in geradem Verhältniss giebt, indem die resp. Proben einer bestimmten Prüfungshitze ausgesetzt werden im Vergleich zu dem Garnkirker Thon, welcher, vermengt mit 1 Theil chemisch reinem Quarz, als Einheit gesetzt ist.

Quantitative Analyse. - $1 \mathrm{Grm}$. des bei $100^{\circ} \mathrm{C}$. getrockneten feinsten Thonpulvers wurde mit kohlensaurem Natronkali aufgeschlossen, und die Kieselsäure, die Thonerde, das Eisen, der Kalk und die Magnesia in bekannter Weise sorgfältig bestimmt. Die Kieselsäure und die Thonerde wurden auf das genaueste ausgewaschen, wobei ich letztere nach $B$ unsen bis zur 20000 fachen Verdünnung 
decantirte. Die Menge der Thonerde musste daher stets eher zu niedrig als zu hoch gefunden werden. - Zur Bestimmung der mechanisch beigemengten Kieselsäure, d. i. des Sandes, wurde 1 Grm. von obigem Thonpulver in einer Platinschale mit überschüssiger, wenig verdünnter Schwefelsäure volle 12 Stunden lang erhitzt, zuletzt bis zum Verdampfen des Hydrats. Alsdann wurde Wasser zugefügt, filtrirt, ausgesüsst, der Rückstand geglüht und gewogen. Letzterer wurde hernach mit überschüssigem kohlensauren Natron 3 bis $4 \mathrm{Mal}$ andauernd gekocht und der ungelöste Theil, der Sand, nach dem Abfiltriren gut ausgewaschen, geglüht und gewogen. - Ferner wurde dio vorher erhaltene saure Lösung zur Bestimmung der Alkalien benutzt, nachdem durch wiederholtes Eindampfen der stets angesäuerten Flüssigkeit und wiederholte Fällung mit neutralem kohlensauren Ammoniak die beigemischten Erden gänzlich entfernt waren. - - Die Menge des Eisenoxyduls wurde durch Digeriren des Thonpulvers mit Salzsäure bei abgeschlossener Luft und nachheriges Titriren mit Chamäleon bestimmt.

Im Allgemeinen geht aus den in den nachfolgenden Tabellen A und B mitgetheilten Analysen hervor, dass die Zusammensetzungsweise dieser sechs Thone mit etwaiger Ausnahme von Nr. 5 und Nr. 3, eine ähnliche ist.

Hebt man unter den Thonen die beiden strengflüssigsten, Nr. 1 und 2, hervor, so sind sie anch die verhältnissmässig thonerdehalligsten, selbst wenn man (vergl. d der Tabelle B) das Verhältniss der Gesammtmenge der Kicselerde zur Thonerde, abgesehen von der untergeordneten Menge der übrigen Basen und nach Abzug des Glühverlustes, in Betracht zieht. Die Gesammemenge der Kieselsäure ist aber für einen feuerfesten Thon, dessen Werth nach seinem endgültigen Verhalten im Feuer geschätzt wird, schliesslich stets cntscheidend.

Setzt man demzufolgo (vergl. e der Tabolle B) dio Gesammtmenge der Kieselsäuro $=100$, so beträgt die Thonerde bei dem Thon Nr. 1 50,96, bei dem Thon Nr. 2 aber 52,63 und bei dem besten schottischen Thon selbst 81,32 . 


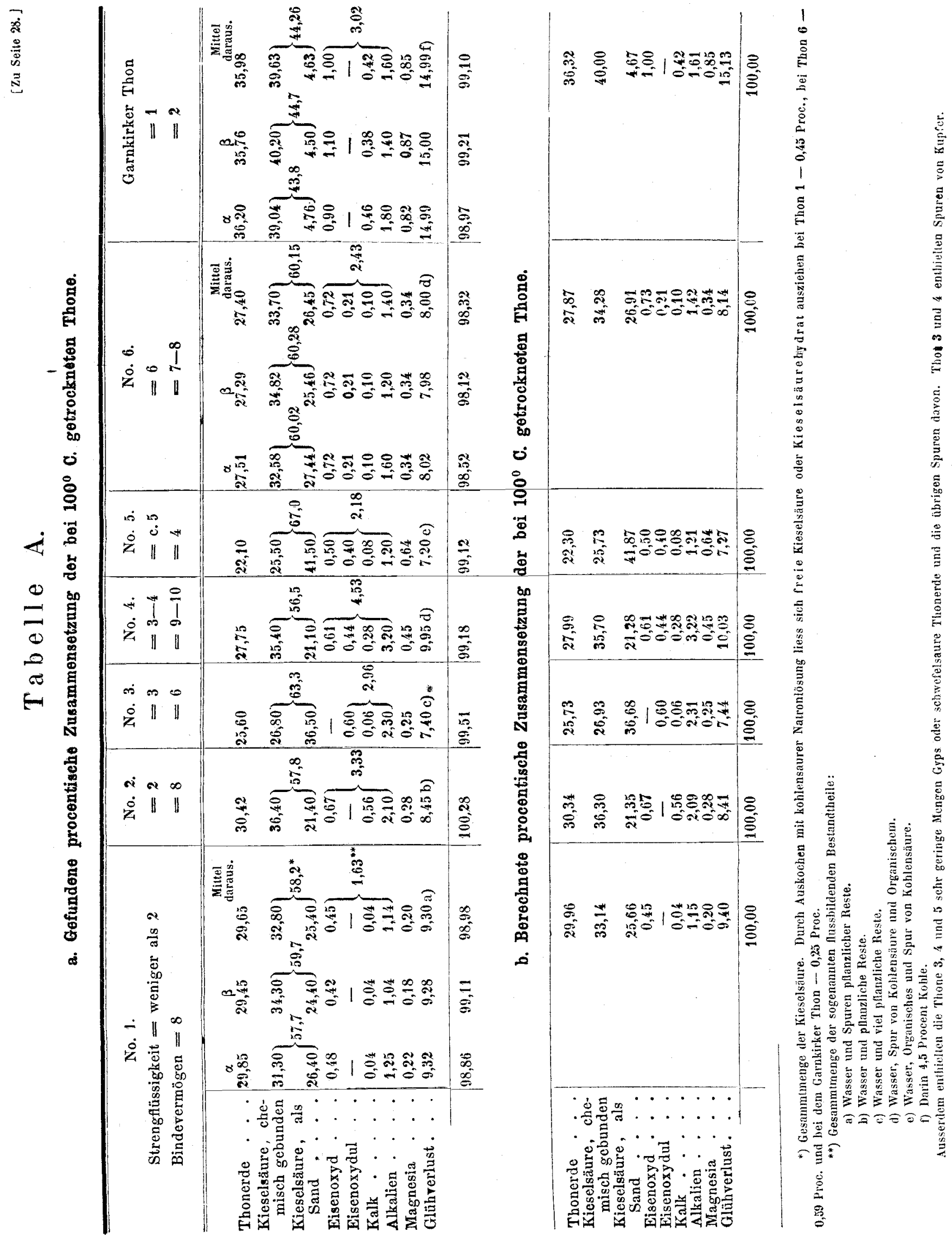




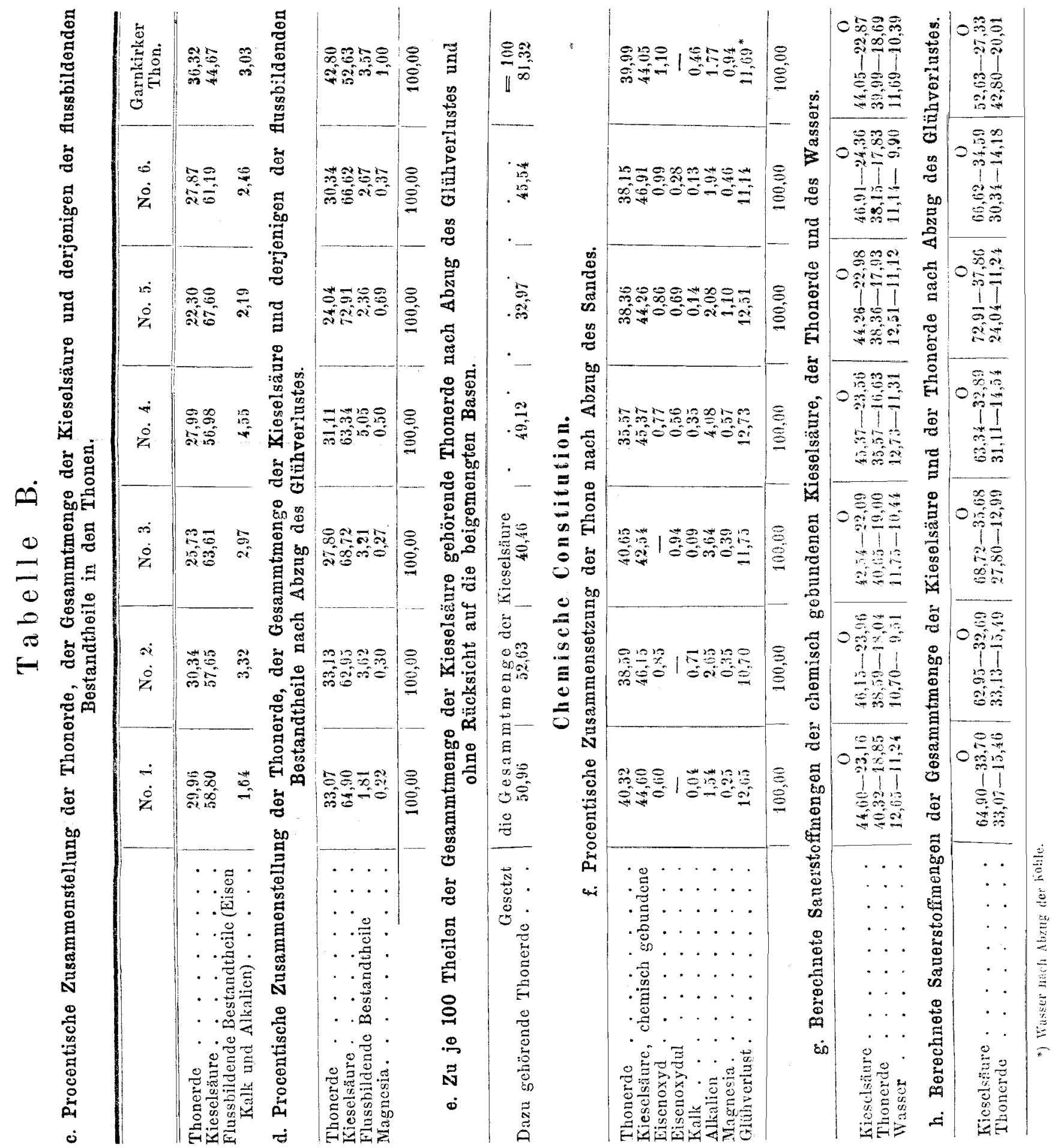


Der Thon Nr. 2, wiewohl er weniger strengflüssig, wenn auch nur um ein Geringes, ist dennoch thonerdehaltiger, eine Ausnahme von dem in Rede stehenden Gesetze, welche aber durch die doppelt so grosse Menge an flussbildenden Basen sich genügend erklären dürfte.

Andererseits steht den übrigen Analysen zufolge kemeswegs die Ab-oder Zunahme der flussbildenden Basen in unbedingtem Zusammenhange mit der Strengflüssigkeit. So enthält gerade der weit strengflüssigste, der Garnkirker Thon, eine grössere Menge an flussbildenden Bestandtheilen, und der leichtflüssigste Thon, Nr. 6, keineswegs dieselben in grösster Menge.

Will man die Zusammensetzung des Thones Nr. 5, der wenig strengflüssig, als Beweis für das aufgestellte Gesetz in umgekehrter Weise gelten lassen, so ist bemerkenswerth, dass er die geringste Menge Thonerde (auf 100 Kieselsäure nur 32,97 Thonerde) enthält.

Um wo möglich herauszufinden, worin zwischen den in ihrer Zusammensetzung im Ganzen sehr ähnlichen, und doch hinsichtlich der Strengflüssigkeit eben so verschiedenen Thonen, Nr. 1 und Nr. 6, die analytischen Kennzeichen liegen, erschien es nicht uninteressant von beiden die Analysen sorgfältigst zu wiederholen, um aus Durchschnittszalilen Schlüsse ziehen zu können.

Da im Ganzen die Resultate für die Thone Nr. 1 und Nr. 6, die beide genau in derselben Weise analysirt wurden, annähernd stimmen, so ist es bei Annahme einer wenigstens relativen Zuverlässigkeit gestattet, folgende sich darbietende vier Gesichtspunkte aufzustellen:

1) Bei dem Thon Nr. 1 findet sich mehr Thonerde als bei dem Thon $\mathrm{Nr}$. 6, und zwar in absoluter wie relativer Hinsicht.

2) Bei dem Thon 1 findet sich weniger Sand mechanisch beigemengt.

3) Die Menge der flussbildenden Bestandtheile ist bei dem Thon 1 eine geringere.

4) Der Wessergehalt ist bei dem Thon 1 ein grösserer. Die Menge der freien Kieselsäure oder der durch kochende Sodalösung ausziehbaren ist bei Thon 1 eine geringere als 
bei Thon 6 , noch weniger beträgt sie bei dem Garnkirker Thon.

Will man diese Resultate, die für den belgischen Thon und solche, die ihm ähnlich, maassgebend sein dürften, allgemein ausdrücken, so lässt sich daraus folgern:

Von zwei oder melveren Thonen, die übrigens emander in der Zusammensetzung sehr almlich, ist derjenige der strengflüssigere, welcher

1) der thonerdehaltigere,

2) am wenigsten Sand mechanisch beigemengt enthäll;

3) wird der strengflussigere auch weniger flussbildende Bestandtheile enthalten, doch ist dabei zu beachten, dass deren nachtheilige Wirkung eine qualitativ verschiedene, und

4) dürfte der grössere Wassergehalt auf eine grössere Strengflüssigkeit deuten *).

Um nicht den Vorwurf der Einseitigkeit oder Voreiligkeit zu verdienen, wovor ich mich um so mehr bewahren möchte, je grösser die Verschiedenartigkeit des Vorkommens der Thone, wie deren Zusammensetzung und Eigenschaften, behalte ich mir ausdruicklich vor, die genannten Ergebnisse noch weiter und umfassender zu verfolgen.

Soll eine Formel fǜ vorstehende Thone aufgestellt werden und vergleicht man zu dem Zwecke die Sauerstofmengen zwischen Kieselsäure, Thonerde und Wasser, und zwar nach Abzuz des Sandes, so ergeben sich die unter lit. $\mathrm{g}$ der Tabelle $\mathrm{B}$ angeführten und bezeichneten Zahlen.

Setzt man die Sauerstoffmenge der Kieselsäure $=4$, so erhält man für den Thon

$\begin{array}{lccc} & \text { Kieselsäure. } & \text { Thonerde. } & \text { Wasser. } \\ \text { Nr. 1. } & 4 & 3,24 & 1,94 \\ \text { Nr. 2. } & 4 & 3,00 & 1,59 \\ \text { Nr. 3. } & 4 & 3,40 & 1,89 \\ \text { Nr. 4. } & 4 & 2,84 & 1,92 \\ \text { Nr. 5. } & 4 & 3,12 & 1,93 \\ \text { Nr. 6. } & 4 & 2,92 & 1,63 \\ \text { Garnkirk Thon } 4 & 3,27 & 1,82\end{array}$

*) In Betreff des Sandgehaltes oder der Kieselsäure überhaupt, dieses nach $H$. Rose in seinen Eigenschafien mehrfach räthsclhaften Körpers giebt es einige Erscheinungen, die auch in feuerfester Hinsicht auf eine gewisse Abhängigkeit von den verschiedenen Zuständen der Kiesclsäure, dem amorphen oder krystallinisehen, deuten. 
Es dürfte hiernach das Verhältniss des Sauerstoffs der Kieselsäure und desjenigen in der Thonerde sein $4: 3$ oder 12:9, und die kieselsaure Thonerde ist somit in diesen Thonen eine basische und hat die Formel $3 . \mathrm{Al}_{2} \mathrm{O}_{3}, 4 . \mathrm{SiO}_{3}$.

Nimmt man die Sauerstoffmenge im Wasser zu $\frac{2}{3}$ der in der Thonerde enthaltenen an, so erhalten wir demnach die Formel

$$
\text { 3. } \mathrm{Al}_{2} \mathrm{O}_{3}, 4 . \mathrm{SiO}_{3}+6 . \mathrm{HO}
$$

was genau entspricht der von Brogniart und Malaguti für den Kaolin aufgestellten Formel.

Bekanntlich fand Fresenius bei seinen ausgezeichneten Untersuchungen von fünf nahe bei einander vorkommenden Thonen aus dem Nassauischen, dass der reine Thon neutrale kieselsaure Thonerde, während Forchhammer und A. Terreil gleichfalls eine basische Formel

aufstellten

$$
\left(2 . \mathrm{Al}_{2} \mathrm{O}_{3}, 3 . \mathrm{SiO}_{3}+6 . \mathrm{HO}\right)
$$

Es bestehen demnach bis jetzt drei bis vier verschiedene Formeln für die Thone, welche dem Vorkommen dieses ungemein, namentlich durch alle sedimentären Formationen bis in die jüngsten Alluvionen verbreiteten sehr verschiedenartigen, bald mehr bald weniger fortgeschrittenen Endzersetzungsproductes, entsprechen dürften.

Fasst man die Sauerstoffmengen der Gesammimenge der Kieselsäure zur Thonerde, nach $\Delta$ bzug des Glühverlustes, ins Auge - ein Verhältniss, das, wenn auch nicht zur Aufstellung einer Formel berechtigt, doch im Feuer endgültig maassgebend ist - so ergeben sich die sub lit. $h$ der Tabelle berechneten Zahlen.

Setzt man die Sauerstoffmenge der Kieselsäure $=2$, so erhält man:

$\begin{array}{lcc} & \text { Kieselsäure. } & \text { Thonerde } \\ \text { Nr. 1. } & 2 & 0,92 \\ \text { Nr. 2. } & 2 & 0,96 \\ \text { Nr. 3. } & 2 & 0,74 \\ \text { Nr. 4. } & 2 & 0,88 \\ \text { Nr. 5. } & 2 & 0,58 \\ \text { Nr. 6. } & 2 & 0,82 \\ \text { Garnkirk Thon } & 2 & \mathbf{1 , 4 3} \\ \text { oder } & \mathbf{3} & \mathbf{2 , 1 3}\end{array}$


Im Allgemeinen ergiebt sich hieraus, mit Ausnahme des Garnkirker Thons, dass wir es schliesslich im Feuer mit nentraler oder gar saurer kieselsaner Thonerile zu thun haben, woraus sich erklärt und gerade beweisen lässt, warum wir die genannten belgischen Thone im Gegensatze zu dęn Steinkohlenthonen niclit zu den ausgezeichnetsten feuerfesten Thonen rechnen können.

Die strengflüssigsten Thone, Nr. 1 und 2, bilden, besonders wenn wir den übrigen Basen einen Theil der Kieselsäure zumessen, alle Kieselsäure mit der Thonerde in chemischer Verbindung gedacht, wenigstens neutrale Verbindungen.

Bei dem schottischen Thone haben wir selbst in diesem Falle ganz entschieden eine basisch-kieselsaure Thonerde vor uns, eine Erklärung für die einzige Vorzüglichkeit dieses schottischen Thones in feuerfester Hinsicht, und gleichzeitig ein Beweis für das aufgestellte Gesetz, der nicht gering in die Wagschale fallen dürfte.

Versucht man aus den verschiedenen, in der Literatur sich vorfindenden Analyson von feuerfesten Thonen das gewonnene Resultat darzuthun, so erscheint hier in der That Thür und Thor geöffnet für jedwede fasl belicbige Behauptung.

Wir finden unter den als höchst feuerfest gerühmten Thonen die allerverschiedenartigste Zusammensetzungsweise, namentlich was das relative Verhältniss zwischen der Kiesel- und Thonerde angeht, wovon erstere jedoch im Ganzen einer meist vorherrsehenden Bestimmung sich zu erfreuen scheint. Abgesehon von manchen darunter befindlichen älteren und unsicheren Analysen, gchört nicht nur eine genaue Silicatanalyse, wie allbekannt und häufig auch deren Ausführung, und besonders die schare Scheidung der Kieselerde von der Thonorde, nicht zu den leichten Aufgaben der analytischen Chemie, sondern die Bestimmung der einen auf Kosten der anderen, ist wohl abhängig von der Uebung des Analytikers. Ferner sind es noch andere Umstände, die das Auffinden einer Gesetzmässigkeit unter dieser wirklichen und scheinbar noch grösseren Verwirrung, erschweren.

Das Beurtheilungsmaass für die feuerfesten Thone ist ein ausserordentlich varïrendes. Die gestellten Anforderun- 
gen sind höchst verschiedenartig und je nach den Umständen, unter denen ein Thon geprüft worden ist, fällt die Entscheidung sehr wechselnd aus. Anderntheils ist die Verschiedenartigkeit der Thone selbst in demselben Lager meist gross genug, um beliebige Proben, je nach dem leitenden Vorurtheil entnehmen zu können, und mag daher auch der Zufall seine Rolle mitspielen.

Ich werde mich daher auf berühmte und allgemeiner bekannte feuerfeste Thone oder auch Fabrikate daraus beschränken, worunter von bewährten Analytikern untersuchte sind.

Vorerst hebe ich die Analyse eines englischen feterfesten Steines von Fresenius hervor.

Die Analyse 1) der Zusammensetzung des ganzen Steines und 2) der darin enthaltenen Chamotte ergab:

\begin{tabular}{lrr} 
& 1, & \multicolumn{1}{c}{2} \\
Kieselsäure & $\mathbf{5 4 , 6 3}$ & \multicolumn{4}{c}{$\mathbf{7 7 , 9 8}$} \\
Thonerde & $\mathbf{4 0 , 2 7}$ & $\mathbf{4 6 , 9 4}$ \\
Eisenoxyd & $\mathbf{2 , 6 7}$ & 2,94 \\
Kalk & $\mathbf{1 , 5 3}$ & $\mathbf{2 , 3 2}$ \\
Magnesia & $\mathbf{1 , 0 3}$ & Spur \\
\cline { 2 - 3 } & $\mathbf{1 0 0 , 1 3}$ & 100,18
\end{tabular}

Berechnet man aus beiden Bestimmungen die Sauerstoffmenge der Kieselsäure und der Thonerde, so ergiebt

$$
\text { 1. } 0 \text { 2. } 0
$$

Kieselsäure $\quad 54,63-28,37 \quad 47,98-24,91$

$$
\text { Thonerde } 40,27-18,82 \quad 46,94-21,94
$$

Ohne allen Zweifel bestand demnach der zur Grundmasse dieses Steins (wovon mir zufällig Stücke zu Händen gekommen sind, welche denselben als ausgezeichnet strengflüssig, gleich den bestbekannten schottischen feuerfesten Steinproben erwiesen) und noch mehr der zur Chamotte verwendete Thon aus basisch-kieselsaurer 'Thonerde.

Unter den besten feuerfesten Thonen Englands und Schottlands finden sich überhaupt mehrere basische Thonsilicate.

So enthalten 1) der Thon von Stannington bei Sheffield, analysirt a) von Le Play und b) von Humbly, 2) von Brierley Hill, Staffordshire, erste Qualität, analysirt von T. H. Henry, 3) von Poole, Dorsetshire, analysirt von Weston, 4) der Chinathon, Koalinit aus Cornwall, analysirt 
von R. A. Cooper, 5) von Stourbridge, analysirt a) von Le Play und b) von Salvetat, und 6) von Grangemouth, analysirt von Penny. Sorte a.

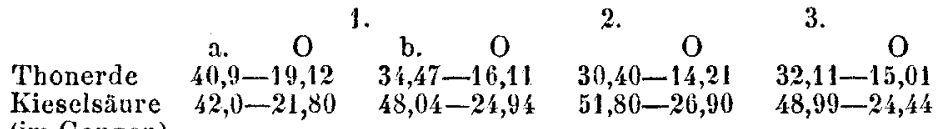
(im Ganzen)

4.

Thonerde $\quad 39,74-18,58$

Kieselsäure $\quad \mathbf{4 6 , 3 2 - 2 4 , 0 5}$ (im Ganzen)
5.

a. 0
$\mathbf{3 8 , 8}-18,14$
$\mathbf{4 6 , 1 - 2 3 , 9 1}$ b.

$28,77-13,45$

$45,25-23,49$
6.

$O$

$35,65-16,66$

$62,8 \tilde{\mathbf{j}}-32,63$

Da die doppelte Sauerstoffmenge der Kieselsäure im Gegensatz zu derjenigen der Thonerde der neutralen Verbindung entspricht, so gehören sämmtliche genannte Thone, ungeachtet der Angabe der Kieselsäure nur in der Gesammtmenge, zu den mehr oder weniger basischen.

Unter den belgischen Thonen besteht der von Maizerouille, welcher wegen seiner grossen Strengflüssigkeit besonders geschätzt wird, analysirt von Coste, ans:
Thonerde
$33,0-15,42$
Kieselsäure (im Ganzen) 56,0-29,08

Unter bekannteren Thonen enthielt der hessische Thon von Gross-Almerode, analysirt a) von Berthier und b) von Salvetat:

Thonerde

a. $\quad 0$

b. $\quad 0$

Kieselsäure (im Ganzen) 46,5-24,14 47,50-24,66

Ferner der Fassaucr Thon, analysirt a) von Forchhammer, b) von Fuchs und c) ron Salvetat:
Thonerde $\quad 33,56-15,69 \quad 35,93-16,80 \quad 28,10-13,14$
a.
b.
$O$
c.
Kieselsäure $\quad 43,30-22,48 \quad 43,65-22,66 \quad 45,79-23,77$ (im Ganzen)

Ferner unter den böhmischen Thonen die Porcellanerde von Gieshübl bei Carlsbad, analysirt von Joh. Czjzek im k. k. polytechnischen Institute zu Wien:

$$
\begin{array}{ll}
\text { Thonerde } & 37,99-17,76 \\
\text { Kieselsäure (im Ganzen) } & 47,50-\mathbf{2 4 , 6 6}
\end{array}
$$


Unter den französischen Thonen finden sich gleichfalls solche, so die Thone von:

Thonerde Abondant. Montunis Journay.
(Saone u. Loire). $\begin{array}{llll}0 & 0 & 0\end{array}$ $40,0-18,7 \quad 45,0-21,0 \quad 31,0-14,5$ Kieselsäure (im Ganzen) 52,0-27,0 55,0-28,6 53,0-27,5

Schliesslich erwähne ich noch die Analysen verschiedener, wenn auch weniger bekannten Kaoline und eines rothen Thones der Provinz Almeria in Spanien, analysirt von Terreil:

Kaolin Kaolin Gewöhnlicher Schr foiner von Almanzor. von Moabdil. rother Thon. rother Thon. 0 $\mathrm{O}$

Thonerde $\quad 31,07-14,52 \quad 30,13-14,08$ Kiesclsäure $\quad 37,99-19,72 \quad 47,17-24,49$ $35,42-16,56$ (im Ganzen)

A. Terreil bemerkt von dem rothen Thon, dass er sich durch seine Zusammensetzung von den gewöhnlichen Thonen unterscheide; er enthalte viel Thonerde und wenig Kieselerde, daher er im höchsten Grade feuerbeständig sei.

Unter den vielen herrschenden ontgegengesetzten Ansichten, welche der Kieselerde die Hanptrolle beimessen, steht die ausgesprochene vereinzelt da, woran sich nur wenige ähnliche, so von Leschen und Andeutungen von Anderen, anschliessen.

Verhältnissmässig kommen demnach unter den wegen der ausgezeichnetsten Feuerfestigkeit sehr gesuchten englischen Thonen die meisten basischen Thonsilicate vor; wir finden jedoch auch solche, und gerade unter den berühmten, in Deutschland, in Böhmon, in Belgien, in Frankreich ete.

In der Gusstahlfabrikation, welche im Allgemeinen die höchsten Anforderungen an die feuerfesten Thone stellt, sind es vornehmlich die, angefülırten Thone oder diesen ähnliche, welche man aufsucht, und es steht erfahrungsmässig fest, dass diejenigen, welche sandhaltig verwerflich sind.

Ueberhaupt, gehen wir die im Handel vorkommenden Thone durch, so sind die fetten, d. h. die in der Regel thonerdehaltigeren, auch die gesuchteren, und häufig besteht der Unterschied in der ersten und zweiten Sorte eines Thones nur darin, dass letztere mehr Sand und resp. mehr 
Kieselerde enthält. Auch in einigen Preiscouranten von Fabriken feuerfester Steine sind es die* reinen Chamottsteine zum Unterschiede von solchen aus sandhaltigem Material, welche für die Anwendung in den grössten Hitzegraden besonders empfohlen werden.

Die bekannte Thonverbesserung, nicht blos in plastischer, homogener, sondern auch in feuerfester Hinsicht, durch Einsumpfen und noch mehr die sogenannte Fäulung (mittelst Jauche und Moorwasser), wobei nach den evidenten analytischen Bestätigungen von Vohl die Alkalien sowohl, wie ein grosser Theil der Kieselsäure in löslicher Form austreten, also die Thone thonerdereicher werden, erklärt sich mit Hülfe des gefundenen Gesetzes überraschend einfach.

Eine Verminderung des Alkaligehaltes, sofern derselbe Procente beträgt, ist jedenfalls in Betracht zu ziehen; jedoch eine gleichzeitige relative Anhäufung der Thonerde durch organische Zersetzungsprocesse ist ein günstiger Umstand, welcher bei der Bildung der Thone der Steinkohlenformation, den unbestritten strengflüssigsten, wirksam gewesen sein möchte.

Für die Annahme, dass manche Thone aus einem Gemenge von Thonerdesilicat und Thonerdehydrat bestehen, sprechen einige analytische Erfahrungen. Der grosse Wassergehalt mancher 'Thone und die vérschiedene Zersetzbarkeit derselben durch Salzsäure ist bekannt; beide Verhältnisse dürften zunehmen mit dem Gehalte an Thonerdehydrat, und in der That scheint es im Ganzen, dass die strengfüssigeren Thone die wasserhaltigeren und die zersetzbareren sind.

Vergleichen wir im Mineralreiche die Schmelzbarkeit der krystallisirt vorkommenden Thonsilicate unter sich, so sind die thonerdehaligsten auch die strengflüsigsten, und zwar in zunelmendem Verhültniss mit dem grösseren Thonerdegehalte.

Hierher gehören der Andalusit, Cyanit, Chiastolith und Diaspor und einige ähnliche nur sehr vereinzelt vorkommende Mineralien. 
Bekannt ist die Unschmelzbarkeit des Cyanits vor dem Löthrohr. Solcher vom Greiner in Tyrol, der nach der Analyse von Jacobson aus

$$
\begin{array}{lr}
\text { Thonerde } & 62,60 \\
\text { Kieselsäure } & 37,30 \\
\text { Eisenoxyd } & 1,08 \\
\hline & 100,98
\end{array}
$$

besteht, der bestimmten Gusstahlschmelzhitze ausgesetzt, hielt sich ohne Zeichen von Schmelzung. Wurde die Temperatur höher gesteigert, so begann die Probe mit Beibehaltung der Form ein wenig zusammenzufritten, und erst bei noch grösserer Steigerung des Hitzegrades bis zur völligen Schmelzhitze des Schmiedeeisens (wie oben controlirt) begann die Masse porcellanartig dicht zu werden. - Als man den Cyanit längere Zcit mit Salzsäure digerirte, wurde Thonerde mit einer geringen Menge Eisen nebst Kalk ausgezogen, und die Strengflüssigkeit schien noch ein wenig zugenommen zu haben.

Etwas weniger strengflüssig verhält sich der Andalusit, wovon eine Probe des Vorkommens auf der Lisenzer Alp in Tirol, analysirt von $B$ unsen, geprüft wurde. Bunsen fand:

$$
\begin{array}{lr}
\text { Thonerde } & \mathbf{5 8 , 6 2} \\
\text { Kieselerde } & 40,17 \\
\text { Manganoxyd } & 0,51 \\
\text { Kalk } & 0,28 \\
\hline & \mathbf{9 9 , 5 8}
\end{array}
$$

In Gusstahlschmelzhitze hielt sich derselbe olne Zeichen von Schmelzung, aber in der Schmelzhitze des Schmiedeeisens sinterte die Probe zur dichten, porcellanartigen, sclineeweissen Masse zusammen mit Beibehaltung der Formen. Die Probe wurde der Mitte eines Krystalls, mit Entfernung der anhaftenden äusseren Glimmerblättchen entnommen.

Als man sie mit Salzsäure längere Zcit digerirte, wurde nicht wenig Thonerde mit eincr geringen Menge Eisen nebst Kalk ausgezogen, wodurch dic Strengflüssigkeit nicht zugenommen hatte.

Ganz ähnlich dem Andalusit verhält sich der Chiastolith. 
Am strengflüssigsten, in Uebereinstimmung mit dem grössten Thonerdegehalte, zeigte sich der Diaspor, und zwar der vorher durch Salzsïure gereinigte.

Zu meinen Versuchen diente eine Probe aus der Gegend von Katharinenburg am Ural, der nach der Analyse von $\mathrm{Dufrenoy}$ besteht aus:

$\begin{array}{lr}\text { Thonerde } & \mathbf{7 4 , 6 6} \\ \text { Wasser } & 14,58 \\ \text { Eisenoxyd } & 4,51 \\ \text { Kieselsäure } & 2,90 \\ \text { Kalk } & 1,64 \\ & 98,29\end{array}$

Als das Mineral, welches schon durch seine starkgelbe Farbe den Eisengehalt zu erkennen giebt, der Gusstahlschmelzhitze ausgesetzt wurde, hiclt sich die Masse in Ganzen ohne Zeichen von Schmelzung; doch liessen sich, unter der Lupe betrachtet, zahlreiche kleine Flusspünktchen wahrnehmen. Wurde durch längeres Digeriren mit Salzsäure das Eisen entfernt nebst einer geringen Menge Thonerde und Kalk, so hielt die weiss gewordene Probe die Schmelshitse des Schmiedeeisens aus olne Zeichen von Schmelzung. Aussen war die Probe, wie auf dem Bruche noch körnig, erdig, und verhiclt sich fast gleich der gleichzeitig mitgeglühten reinen Thonerde.

Verglichen mit dem reinen Mono-Thonerdesilicat, verhält sich schon der Andalusit strengflüssiger, und um so mehr der Cyanit und der gereinigte Diaspor.

Das Ergebniss vorstehender gesammter Versuche und Belege dürfte demnach, kurz ausgedrückt, sein:

Das Wesen der Feuerbeständigkeil ier Thone besteht in deren Thonerdegehalt.

Einzig diese Beantwortung der gestellten Aufgabe ergiebt sich nothwendig, wenn wir uns, von dem bisherigen, vornehmlich relativen, oder einem gar cinseitigen Standpunkte absehend, auf einen meln absoluten und allgemeinen stellen.

Ist z. B. die Behauptung, dass durch Kieselsäurezusatz die Strengflüssigkeit eines Thones zu erhöhen sei, eine nicht unrichtige, so hat sie doch nur eine durchaus relative Gültigkeit, d. h. nur für so lange ist sie geltend, als der 
Braun: Gelbes u. weisses Hydrat der Wolframsäure.

Beurtheilende keinen wesentlich höheren Hitzegrad als Gusstahlschmelzhitze anwendet.

Einleuchtend ist, dass für die Praxis und deren Zwecke in vielen Fällen dieser relative Standpunkt genügt, wozu häufig noch ökonomische, locale oder gewisse technischpraktische Verhältnisse als bestimmend kommen, und daher andere Fragen maassgebend sind. Die technisch-praktische Frage, wie verhalten sich die basischen Thonsilicate im Gegensatze zu den neutralen und den sauren, zu anderen Flüssen, ist gleichfalls, wie wichtig sie auch ist, eine relative.

Modificiren ferner die sogenannten flussbildenden Bestandtheile die Güte eines feuerfesten Thones, so sind sie nur in beschränkter Weise entscheidend.

Fragt man dagegen absolut und abgesehen von nur relativen Beschränkungen, welches Thonsilicat hält den höchsten und andauerndsten Hitzegrad aus, ohne zu schmelzen, so ist positiv zu antworten: dasjenige, welches den grössten Thonerdegehalt hat.

So ist das basische Thonsilicat strengflüssiger als das neutrale (einfache), und das saure ist leichtflüssiger als das neutrale.

Ehrenbreitstein am Rhein, den 10. August 1863.

\section{IV. \\ Ueber das gelbe und weisse Hydrat der Wolframsäure.}

Von

C. D. Braun.

Vor melireren Jahren hatte icli Gelogenheit, in dem Laboratorium des Herrn Professor Otto in Braunschweig mich mit dem Studium der Wolframsäure zu befassen, und machte schon damals die Beobachtung, dass das weisse 